\title{
Highlights in the hadron electromagnetic structure
}

\author{
Egle Tomasi-Gustafsson ${ }^{1, \star}$, Simone Pacetti ${ }^{2}$, and Andrea Bianconi ${ }^{3}$ \\ ${ }^{1}$ IRFU, CEA, Université Paris-Saclay, 91191 Gif-sur-Yvette Cedex, France \\ ${ }^{2}$ Dipartimento di Fisica e Geologia, and INFN Sezione di Perugia, 06123 Perugia, Italy \\ ${ }^{3}$ Dipartimento di Ingegneria dell'Informazione, Università di Brescia and \\ Istituto Nazionale di Fisica Nucleare, via A. Bassi 6, 27100 Pavia, Italy
}

\begin{abstract}
In frame of a general view of proton electromagnetic form factors, two recent findings related to reanalyses of data are presented. Recent experiments in the scattering and in the annihilation region provided us with more precise data and/or extending the kinematical region, allowing a deeper analysis and a common view of these fundamental quantities. We will discuss two issues: the discrepancy between the form factors extracted from unpolarized and polarized $e p$ elastic scattering experiments, in connection with the commonly used dipole parametrization; peculiar oscillations in $e^{+} e^{-} \rightarrow \bar{p} p(\gamma)$ annihilation cross section, that become periodical when plotted as a function of the 3momentum of the relative motion of the final proton and antiproton, after subtraction of a smooth function.
\end{abstract}

\section{Introduction}

Electromagnetic hadron form factors (FFs) are fundamental quantities that describe the internal structure of non-pointlike particles, their charge and magnetic currents. Assuming that the interaction occurs through one photon exchange, FFs can be measured (through electron proton elastic scattering and $e^{+} e^{-} \leftrightarrow \bar{p} p$ annihilations): in the first case, the transferred photon of virtual mass $Q^{2}=-q^{2}>0$ is space-like, in the second case, the time-like photon has 4-momentum squared $q^{2}>0$. FFs are also directly calculated, as they enter in the expression of the electromagnetic current: hadron models, after reproducing the static properties of hadrons, as masses and magnetic moments, should reproduce their dynamical content, parametrized by FFs. Schematically, FFs at large momentum transfer probe the quark structure of the hadrons whereas at low momentum transfer, they probe the size of the hadron.

The analytical properties of FFs justify a common interpretation of scattering and annihilation experiments. It is tempting to have a global definition of FFs, in the space and time-like regions. In [1] the definition of FFs was generalized as follows:

$$
F\left(q^{2}\right)=\int_{\mathcal{D}} d^{4} x e^{i q_{\mu} x^{\mu}} \rho(x), q_{\mu} x^{\mu}=q_{0} t-\vec{q} \cdot \vec{x}
$$

where $\rho(x)=\rho(\vec{r}, t)$ is the space-time distribution of the electric charge in a space-time volume $\mathcal{D}$.

\footnotetext{
^e-mail: egle.tomasi@cea.fr
} 
In the scattering channel, $e+p \rightarrow e+p$ and in the Breit frame, we recover the definition of FFs as Fourier transform of a charge density:

$$
F\left(q^{2}\right)=\delta\left(q_{0}\right) F\left(Q^{2}\right), Q^{2}=-\left(q_{0}^{2}-|\vec{q}|^{2}\right)>0,
$$

where the zero energy transfer is implied.

In the annihilation channel:

$$
F\left(q^{2}\right)=\int_{\mathcal{D}} d t e^{i \sqrt{q^{2}} t} \int d^{3} \vec{r} \rho(\vec{r}, t)=\int_{\mathcal{D}} d t e^{i \sqrt{q^{2}} t} Q(t),
$$

where $Q(t)$ can be interpreted as the time evolution of the charge distribution in the domain $\mathcal{D}$. In [1] a qualitative phenomenological picture of scattering and annihilation processes was presented, and the possibility of an observable dynamical polarization of the formed hadron pair was suggested.

Hadron FFs are investigated since decades, but recent findings and experimental possibilities have renewed interest in this field. High intensity electron beams, with large and stable polarization, together with large acceptance spectrometers and detectors, and the development of hadron polarimetry allowed in particular to apply to the Akhiezer-Rekalo recoil proton polarization method [2, 3], that requires to measure the recoil proton(neutron) polarization in the scattering of longitudinally polarized electrons. These experiments, performed at MIT Bates (USA), MAMI (Germany) and JLab (USA) at large momentum transfer, brought very precise information on the ratio of the electric to magnetic FF for protons and neutrons and showed that this ratio is not constant, but decreases for increasing momentum transfer approaching zero at $9 \mathrm{GeV}^{2}$. It was previously assumed that the electric and magnetic FFs follow a dipole dependence $\simeq\left(Q^{2}\right)^{-2}$. Still unpolarized $e p$ scattering measurements seem to confirm such behavior. This discrepancy gave rise to a large number of theoretical papers and new experiments. We reanalyze the unpolarized cross section data in terms of FF ratio and show that the discrepancy between sets of data vanishes if one takes into account, besides effects of radiative corrections and parameter correlations, the effect of relative normalization.

The time-like region is accessible at $e^{+} e^{-}$colliders, as BABAR (USA), BESIII (China), and VEPPII (Russia), through the reaction $e^{+} e^{-} \rightarrow \bar{p} p(\gamma)$ and at $\bar{p} p$ colliders as LEAR (CERN) and FermiLab (USA). In next future a high energy and high intensity antiprotons beam will be available at PANDA (FAIR, Germany). The presence of oscillating structures in the time-like FF data has been recently highlighted in $[4,5]$. In particular the data present a regularity when plotted as a function of the relative momentum of the outgoing hadrons. This pattern is tentatively associated with the interference between two sources, respectively of the quark and of the hadron size, bringing the signature of the first instants of the hadron formation from the excited vacuum.

\section{Reanalysis of Rosenbluth data}

It has been assumed for long time that the proton electric FF, as well as the magnetic FFs of the proton and neutron, normalized to their magnetic moment, have a $Q^{2}$-dipole dependence $(Q$ is the four-momentum of the photon):

$$
G_{D}\left(Q^{2}\right)=\left(1+\frac{Q^{2}[\mathrm{GeV}]^{2}}{0.71}\right)^{-2},
$$

whereas the neutron electric FF is essentially zero. The dipole parametrization was commonly accepted, because it was consistent with the non-relativistic approximation, where FFs are Fourier transforms of the spatial densities of electric charge and magnetization of the nucleon; in such frame, the 


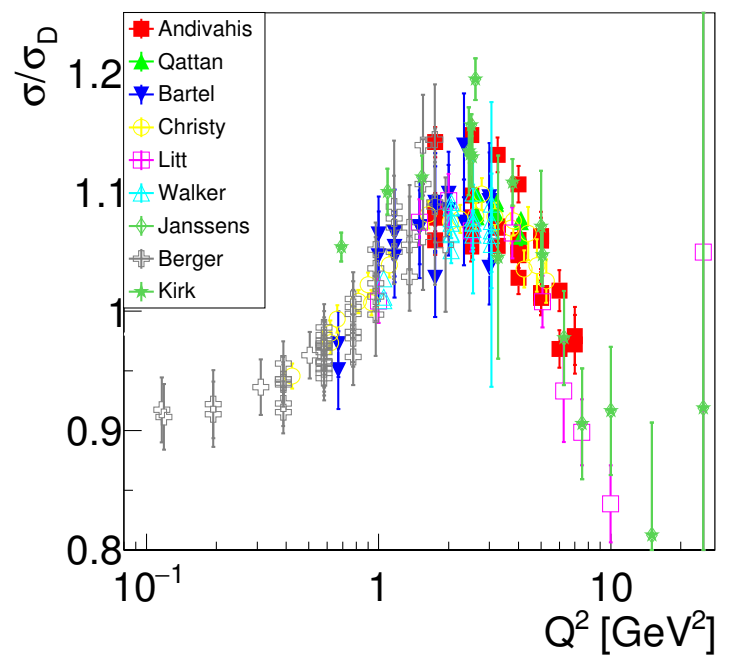

Figure 1. Cross section normalized to the dipole cross section, $\sigma / \sigma_{D}$, as a function of $Q^{2}$ for different experiments.

dipole approximation corresponds to an exponential charge distribution. Moreover, from the QCD point of view, as elastic FFs represent the probability that a proton remains in its ground state after that each of its valence quarks has received a momentum squared, $Q^{2}$, transferred by the virtual photon. Scaling laws predict a $\left(1 / Q^{2}\right)^{2}$ dependence of the amplitude of the process $[6,7]$ (corresponding to two gluon exchange, the minimum number of exchanges needed for sharing the momentum among the three valence quarks).

However, several early experiments pointed out a deviation of the elastic ep cross section from the $\left(1 / Q^{2}\right)^{-5}$ behavior. Quoting a presentation of the data at the highest available transferred momenta, from Nobel prize R. Taylor: "There appears to be definite evidence in the data for a significant deviation from the dipole fit" [8]. The dipole normalized cross section

$$
\frac{\sigma}{\sigma_{D}}=\frac{\sigma_{\mathrm{red}}^{\exp }}{G_{D}^{2}\left(\epsilon / \mu^{2}+\tau\right)},
$$

being $\sigma_{\text {red }}^{\text {exp }}$ the measured reduced cross section, is reported in figure 1 as a function of $Q^{2}$, regardless of the value of $\epsilon$. Here $\tau=Q^{2} /\left(4 M^{2}\right), M$ is the proton mass, $\mu$ is the proton magnetic moment. The $Q^{2}$ coordinates for the data at different $\epsilon$ are seen as vertically quasi-aligned symbols. Note that if these points form a cluster with overlapping error bars, it means that they are compatible with the relation $G_{E} \simeq G_{M} / \mu \simeq G_{D}$. If points are not overlapping, then FFs do not follow a dipole behavior. In general, and particularly at large $Q^{2}$, one can see that the dipole fit is not a good representation of the data. The deviation at large $Q^{2}$ reaches $20-30 \%$ on the cross section and has to be attributed mainly to the magnetic term. The magnetic contribution to the elastic cross section becomes dominant at large $Q^{2}$. The reduced cross section of electron-proton elastic scattering $\sigma_{\text {red }}$, in the one-photon exchange approximation, is linear in the variable $\epsilon=\left[1+2(1+\tau) \tan ^{2}\left(\theta_{e} / 2\right)\right]^{-1}$, being $\theta_{e}$ the electron scattering angle in the proton rest frame:

$$
\sigma_{\text {red }}\left(\theta_{e}, Q^{2}\right)=\left[1+2 \frac{E}{M} \sin ^{2}\left(\theta_{e} / 2\right)\right] \frac{4 E^{2} \sin ^{4}\left(\theta_{e} / 2\right)}{\alpha^{2} \cos ^{2}\left(\theta_{e} / 2\right)} \times \epsilon(1+\tau) \frac{d \sigma}{d \Omega}=\epsilon G_{E}^{2}+\tau G_{M}^{2},
$$

where $E$ and $d \sigma / d \Omega$ are the electron initial energy and the differential cross section in the proton rest frame, and $G_{E}$ and $G_{M}$ are the proton electric and magnetic Sachs FFs. The measurement of 
the differential (reduced) cross section at fixed $Q^{2}$, for different angles, allows to extract the squared values of the FFs, $G_{E}^{2}$ and $G_{M}^{2}$, as the slope and the intercept (multiplied by $\tau$ ), respectively, of this linear distribution (Rosenbluth separation [9]).

The doubts on the deviation from the dipole approximation for the electric FF, became evident with the advent of polarization experiments. In the 70's Akhiezer and Rekalo [2, 3] showed that the polarization of the scattered proton in the scattering of longitudinally polarized electrons on an unpolarized target (or the asymmetry with a transversely polarized target) contains an interference term proportional to the product $G_{E} G_{M}$. This observable would therefore be more sensitive to a small electric contribution, and even to its sign (particularly important for the neutron case). A measurement of the ratio of the transverse to the longitudinal polarization of the recoil proton gives a direct measurement of the ratio of electric and magnetic FFs, $R=G_{E} / G_{M}$ :

$$
\frac{P_{t}}{P_{\ell}}=-2 \cot \left(\theta_{e} / 2\right) \frac{M}{E+E^{\prime}} \frac{G_{E}}{G_{M}},
$$

( $E^{\prime}$ is the scattered electron energy) and it is free from systematic errors coming from the beam polarization and the analyzing powers of the polarimeter. The data based on the Akhiezer-Rekalo method, mostly taken by the JLab GEp collaboration ([10] and references therein), showed with unprecedented precision that the ratio of electric to magnetic FFs decreases as $Q^{2}$ increases. Below it is shown that recent reanalyses of Rosenbluth data in electron proton elastic scattering in terms of the FF ratio, show agreement between the value of this ratio whenever extracted from polarized or unpolarized experiments [11]. In particular the large sensitivity of the ratio to relative normalization of the cross section data solves the discrepancy, within the assumption of the one photon exchange mechanism.

\subsection{Reanalysis of existing data}

Problems of parameter correlations and limits inherent to the Rosenbluth have been discussed in [12]. Previous global analyses were done, discussing in particular the problem of normalization among different sets of data, the omission of some of the data points [13] or reconsidering radiative corrections [14].

The following procedure to extract the FFs information from the unpolarized cross section has been suggested in [11]. Instead of extracting separately $G_{E}$ and $G_{M}$, we write the reduced cross section given in (4) as

$$
\sigma_{\text {red }}=G_{M}^{2}\left(R^{2} \epsilon+\tau\right),
$$

where $G_{M}^{2}$ and $R^{2}=\left(G_{E} / G_{M}\right)^{2}$ are considered as independent parameters. The unpolarized data are fitted at fixed $Q^{2}$. The procedure has the advantage to extract directly the ratio, by automatically accounting for the effect of the correlations between $G_{E}$ and $G_{M}$. The parameter $R^{2}$ represents directly the deviation of the linear dependence of the cross section from a constant $\epsilon$ value, whereas eventual general normalization and systematic errors are absorbed by $G_{M}^{2}$.

If for some of the data values and errors consistent with the original publication are recovered, the data from [15] deserve a specific discussion. This work is especially representative, as it extends the individual FF extraction by the Rosenbluth method to the largest values of $Q^{2}$.

\subsubsection{Data from [15]}

The data of [15], with eight points and two settings, span the region $1.75 \leq Q^{2} \leq 8.83 \mathrm{GeV}^{2}$. The two settings will be indicated as high energy (HE) and low energy (LE) experiments. 


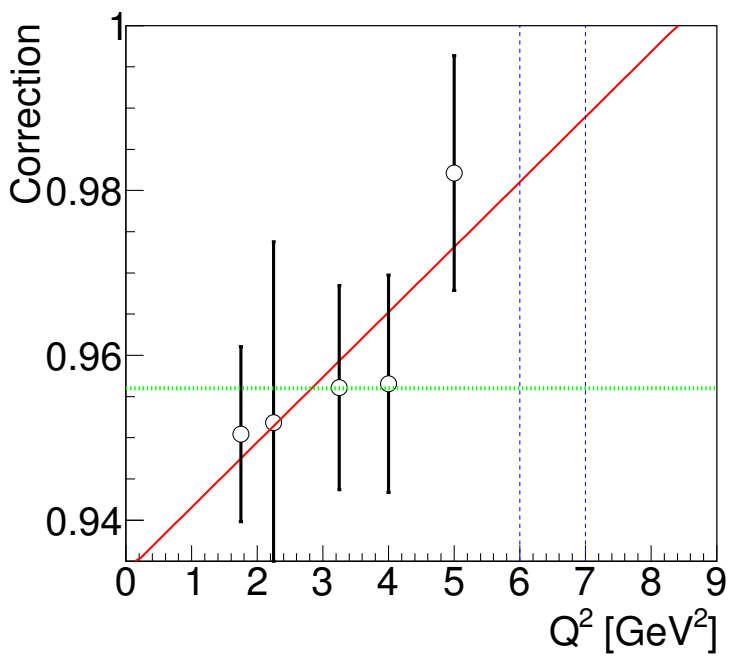

Figure 2. Correction factor as a function of $Q^{2}$. A linear fit (red line) shows an increasing of the factor. The dashed (blue) lines indicate that the extrapolated correction for the two HE points would be close to $1 \%$ instead than $\simeq 5 \%$, as applied in the original paper.

In the original paper the measured cross sections were published, warning that an uncertainty of $\pm 5 \%$ affected the second setting, due to a poor knowledge of the acceptance of the spectrometer. This error, however, was not added to the tabulated error. Instead, it was taken into account as a constant relative correction, fixed on the two lowest values $Q^{2}=1.75$ and $2.50 \mathrm{GeV}^{2}$ (the cross section was measured at each setting for the lowest $\epsilon$ value). The cross section from the LE setting was larger by $4-5 \%$. Assuming a linear $\epsilon$ dependence of the reduced cross section (that implies the dominance of the one-photon exchange mechanism), a fit of the HE data was done and the LE energy point was renormalized to lie on the straight line. Then, the same constant normalization $C=0.956$, fixed on the low $Q^{2}$ point, was applied to the cross section at all $Q^{2}$ (Analysis I). This procedure has the effect to enhance the slope, increasing the FF ratio. Note that for $Q^{2}=6$ and $7 \mathrm{GeV}^{2}$ only two points are present. The renormalization (lowering) of the first point changes essentially the slope of the linear fit.

The data were reanalyzed following several procedures. We recalculated the ratio using the data as published, without renormalizing the two settings and considering the LE points as additional, independent measurements. In this case the data points at $Q^{2}=1.75$ and $2.5 \mathrm{GeV}^{2}$ are both included in the fit, constraining the fit to an average value (Analysis II).

We fit only the HE points (excluding therefore the two points at $Q^{2}=6$ and $7 \mathrm{GeV}^{2}$ ). We find a slope consistent with analysis II, although affected by larger errors, as the number of points is smaller (Analysis III).

We repeat the normalization procedure, by aligning the LE point on the straight line fitting the HE points (Analysis IV). We note a systematic increase of the normalization factor (figure 2). Applying a normalization coefficient that is not constant with $Q^{2}$ but derived in order to align the LE point to the straight line defined by the HE points turns out to be equivalent to Analysis III in terms of slope and intercept.

The results are reported in figure 3 and compared to the ratio from polarization data. We may conclude that the results from Analysis II, III, and IV are consistent with the decreasing of the ratio indicated by the polarization data. Therefore a revision of the normalization factor brings the data into agreement. Moreover, at the light of all above, it is nonsense to use the FFs data from [15] to probe the two-photon effect, as they were extracted under the hypothesis of linearity of the reduced cross 


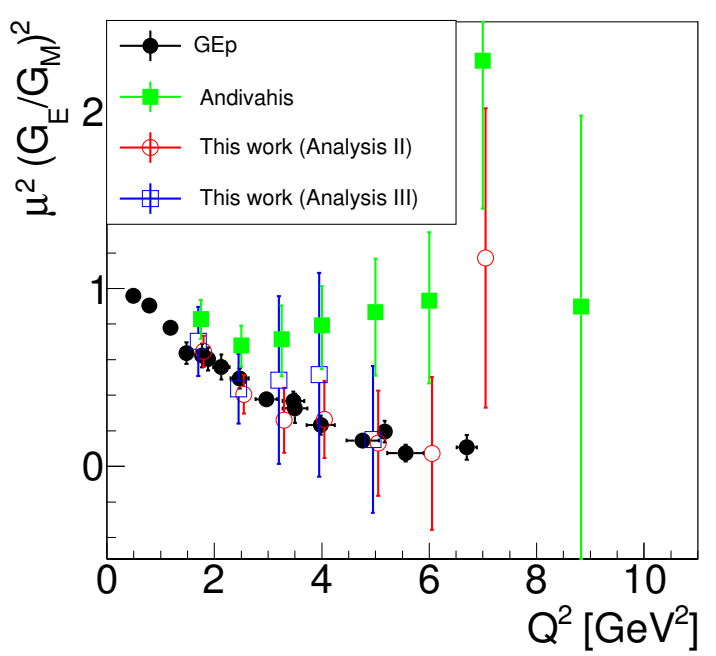

Figure 3. Color online. $\mu^{2} R^{2}=\mu^{2}\left(G_{E} / G_{M}\right)^{2}$ as a function of $Q^{2}$ from (Andivahis: [15], green solid squares) as originally published; from Analysis II: without renormalization (red open circles); from Analysis III: omitting the lowest $\epsilon$ point (blue open squares) compared to the values from polarization experiments (GEp: [10], black solid circles).

section, by correcting the first point to be aligned. The results showed consistency with the hypothesis $\mu^{2} R^{2} \simeq 1$ at large $Q^{2}$, as expected at that time. The tendency of the first two points to deviate from unity was operatively corrected by the renormalization procedure.

Among the available data, three sets [16-18] show a particular behavior, that is not consistent with the previous finding, giving a value of the ratio exceeding unity and growing with $Q^{2}$.

For these experiments it was noted in [12] that radiative corrections and/or correlations are especially large. The data from [18] were extracted detecting the proton instead of the electron. Besides the above mentioned corrections, at large $Q^{2}$ the contamination of the elastic peak by the inelastic $e+p \rightarrow e+p+\pi^{0}$ reaction has to be carefully subtracted [10].

For [16, 17], $G_{M}^{2}$ extracted from the present analysis is systematically lower, showing that these measurements may be affected by a global systematic error probably due to normalization issues, whereas the results of [18] agree with the standard parametrization of the magnetic contribution.

Note that in [17] a somehow arbitrary renormalization was done by "changing the normalization of the small angle data from SLAC or DESY by $\pm 1.5 \%$ with respect to the large angle data (Bonn)". This normalization increased the FFs ratio towards unity.

\section{Periodic oscillations in the time-like region}

In the time-like region, FFs become complex, due to unitarity, and the unpolarized cross section depends on their squared moduli, assuming one photon exchange approximation. The most recent data on electron-positron annihilation into a proton-antiproton pair have been collected by the BABAR collaboration, through initial state radiation (ISR): $e^{+}+e^{-} \rightarrow \bar{p}+p(\gamma)$. When the photon is hard, the cross section can be factorized in a radiator factor, that depends on the hard photon kinematics only, multiplied by the cross section of the process of interest $e^{+}+e^{-} \rightarrow \bar{p}+p$. The recent data by the BABAR collaboration $[19,20]$ cover with reasonable continuity a region ranging from slightly over the $\bar{p} p$ threshold to $q^{2} \approx 36 \mathrm{GeV}^{2}$. In particular about 30 data points have been extracted in the region $q^{2}<10 \mathrm{GeV}^{2}$, with a relative error lower than $10 \%$. Due to the precision and the continuity of these data, oscillations of the cross section as a function of the $q^{2}$ become evident. 
From the cross section, one extracts a generalized FF:

$$
\left|F_{p}\right|^{2}=\frac{3 \beta q^{2} \sigma}{2 \pi \alpha^{2}\left(2+\frac{1}{\tau}\right)}
$$

(where $\left.\alpha=e^{2} /(4 \pi), \beta=\sqrt{1-1 / \tau}, \tau=\frac{q^{2}}{4 M^{2}}\right), M$ is the proton mass.

Plotting the generalized FF as a function of the 3-momentum of the relative motion of the final proton and antiproton, a systematic sinusoidal modulation appears in the near-threshold region, typical of an interference pattern, figure 4 .

Through a Fourier analysis, the period is shown to correspond to a relative distance of $0.7-1.5 \mathrm{fm}$ between the centers of the forming hadrons. It has been suggested that a rescattering process at the level of the newly formed hadrons interferes with the processes at a much smaller scale driven by the quark dynamics [4]. These oscillations may be interpreted as a signature of early mechanism of hadron creation.

We introduce a function of the form

$$
F(p)=F_{0}(p)+F_{o s c}(p),
$$

where $p\left(q^{2}\right)=\sqrt{E^{2}-M^{2}}, E=q^{2} /(2 M)-M$, and $p$ is the momentum of one of the two hadrons in the frame where the other one is at rest. The function $F_{0}(p)$ is a smooth background, expressing the regular behavior of the FF over a long $q^{2}$ range, whereas $F_{\text {osc }}(p)$ describes the overlapping oscillations.

The recent data are best reproduced by the function $F_{0}$ proposed in [21]:

$$
\left|F_{0}\left(q^{2}\right)\right|=\frac{\mathcal{A}}{\left(1+q^{2} / m_{a}^{2}\right)\left(1-q^{2}[\mathrm{GeV}]^{2} / 0.71\right)^{2}}, \mathcal{A}=7.7, m_{a}^{2}=14.8 \mathrm{GeV}^{2} .
$$

The best fit function is then subtracted from the data, leaving a regular oscillatory behavior, figure 4 . It has magnitude $\sim 10 \%$ of the regular term, and is well visible over the data uncertainties for $p>3 \mathrm{GeV}$. We have fitted the difference between the BABAR data and the regular background term $F_{0}(p)$ with the four-parameter function

$$
F_{\text {osc }}(p)=A e^{-B p} \cos (C p+D) .
$$

This difference is plotted in the lower panel of figure 4 . The values of the parameters are, respectively, $A=0.05 \pm 0.01, B=(0.7 \pm 0.2) \mathrm{GeV}^{-1}, C=(5.5 \pm 0.2) \mathrm{GeV}^{-1}$, and $D=0.0 \pm 0.3$. The relative errors in the parameters $C$ and $B$ show that the oscillation period is better defined than the damping coefficient $e^{-B p}$. Two and a half oscillations are clearly visible over the reaction threshold, while for $p>2.8 \mathrm{GeV}$ the vertical error bars overcome the oscillation amplitude $A e^{-B p}$.

The parameter $D$ defines the position of the first oscillation maximum that occurs at $p=0$ within the error $\Delta D P /(2 \pi)$, where $P$ is the oscillation period. Estimating the oscillation period $P=1.13$ $\mathrm{GeV}$, the first oscillation maximum occurs at $p=0$ within an error of $0.05 \mathrm{GeV}$.

Since $p$ is a variable that is uniquely associated with the relative motion of the hadron, periodicity is associated to interactions (rescattering) between the forming hadrons after the virtual photon has been converted into quarks and antiquarks.

Let $\vec{r}$ be the space variable that is Fourier-conjugated to $\vec{p}: r=|\vec{r}|$ is the distance between the centers of mass of the two forming hadrons, in the frame where one is at rest. The observed behavior has been modeled via a two-stage process [22] where: 1) a $\bar{p} p$ pair is formed at a distance $r$ with space distribution amplitude $M_{0}(r) ; 2$ ) rescattering takes place between the newly formed hadrons ( $p$ and $\bar{p}$ ) according to an optical potential that is function of their distance $r$. Rescattering was modeled via the 


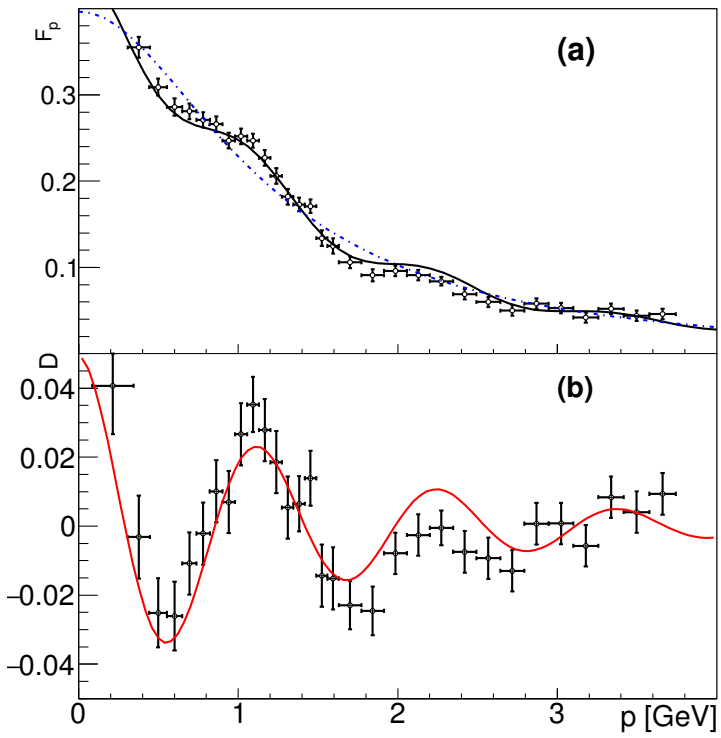

Figure 4. a) FF data from $B A B A R$ as a function of $p_{L a b}$, global fit: black solid line. b) after subtraction of a background function (blue dotted line), damped regular fit function (red solid line).

Distorted Wave Impulse Approximation (DWIA) formalism, following the scheme employed in [23]. The starting point is the Fourier transform

$$
F_{0}(p)=\int d^{3} \vec{r} e^{i \vec{p} \cdot \vec{r}} M_{0}(r)
$$

where we interpret $e^{i \vec{p} \cdot \vec{r}}$ as the plane wave final state of the $\bar{p} p$ pair in their center of mass, and $M_{0}(r)$ as a matrix element describing the earlier stage of the process. Plane Wave Impulse Approximation (PWIA) corresponds to the absence of rescattering: $\psi_{f}(\vec{r})=e^{i \vec{p} \cdot \vec{r}}$. In the distorted DWIA formalism a simple factorized distortion $D(\vec{r})$ is introduced:

$$
\psi_{f}(\vec{r})=D(\vec{r}) e^{i \vec{p} \cdot \vec{r}}
$$

where $D(\vec{r})$ is calculated as a Glauber-like eikonal factor:

$$
D(x, y, z)=e^{-i b \int_{z}^{\infty} \rho\left(x, y, z^{\prime}\right) d z^{\prime}},
$$

where $\hat{z} / / \vec{p}$ and $b$ is a complex number. When $b$ is real, the rescattering potential is elastic, and it is attractive or repulsive (depending on the relative sign of $\operatorname{Re}(b)$ and $p_{z}$ ), when $b$ is pure imaginary, the potential generates flux absorption or flux creation. The origin of an optical potential with an imaginary part is related, in general, to the fact that a multi-channel process is inclusively projected onto one channel alone.

After testing several configurations, our best results have been obtained with double-layer imaginary potentials, presenting two $r$-ranges where the potential phase is opposite, i.e., they present an inner region where the $\bar{p} p$ flux is produced and an outer region where the $\bar{p} p$ flux is absorbed, as illustrated in figure 5. All of them are purely imaginary $(\operatorname{Re}(b)=0)$. The former two potentials have been calibrated to reproduce, as well as possible, BABAR data.

These potentials have been able to produce periodic oscillations with a period of $1 \mathrm{GeV}$ or shorter, and of arbitrarily large amplitudes, depending on the parameters [24]. To reproduce LEAR elastic and annihilation data, phenomena taking place at small $r$ have no relevance, since the surface interaction 


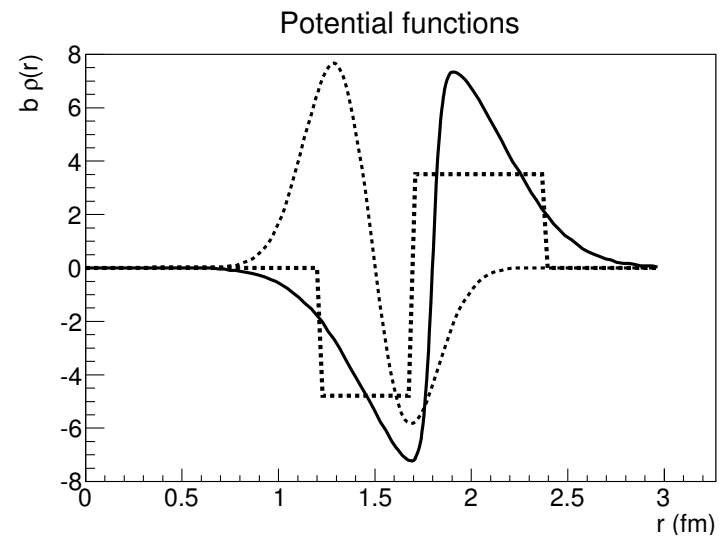

Figure 5. Three double-layer potentials used in (13) to calculate the final state distortion factor $D(x, y, z)$. They may reproduce the FFs data (background and oscillations), with an opportune choice of the parameters.

at $r \approx 1-2 \mathrm{fm}$ prevents most of the initial $\bar{p} p$ channel wave function from entering the $r<1 \mathrm{fm}$ region. The "regeneration" effect due to the inner potential introduced above would have little effect on the total elastic and inelastic cross sections, since it affects only a very small component of the wave function.

On the other side, this small component which is nonzero at small $r$ is essential for the coupling of the proton-antiproton pair with a virtual photon with virtuality $q^{2}>4 M^{2}$. The coupled regeneration/absorption mechanism produces, for $r<2 \mathrm{fm}$, an alternance of regions where this component of the $\bar{p} p$ wave function is enhanced or suppressed.

Specific conditions are necessary to reproduce the observed effect. An imaginary optical potential that is mainly flux-generating in a region of small distances between the centers of the forming (and still overlapping) proton and antiproton, and mainly flux-absorbing at larger distances, produces systematic oscillations of the effective proton TL FF, consistent with the observed ones. At distances $\approx 1-2 \mathrm{fm}$ this potential behaves as the optical potentials ordinarily used to reproduce $\bar{p} p$ annihilation data: it damps the $\bar{p} p$ flux by annihilating $\bar{p}$ and $p$ into multi-meson states. A possible explanation for the regeneration features of the potential at smaller distances could be in terms of coupling between the $\bar{p} p$ final channel and large-mass virtual states (like baryon-antibaryon) temporarily produced by the virtual photon. In order to reproduce the data, the transition from the flux-generating to the fluxabsorbing region must be sudden.

\section{Conclusion}

Form factors data in the space and time-like regions have been critically revised and reanalyzed. In the space-like region, the conclusion is that no real discrepancy exists between the FF extraction from polarized and unpolarized experiments, once all corrections (radiative, normalizations, correlations) are taken carefully into account. There is no need to claim for a large contribution of two-photon exchange. The presence of such contribution (beyond the expected $\alpha$-counting size) would make the formalism of $e p$ scattering experiments very involved, and invalidate most of the information of the nucleon structure extracted so far.

In the time-like region, precise data open the possibility to access detailed features of the inner structure of the proton, and to shed light on the non-perturbative and pertubative aspects of the creation of matter from the quantum vacuum created by the annihilation process. 
The perspectives of a global phenomenological frame and of new, precise data to be collected in next future open the way to access the details of the hadron formation and of the inner composition of the hadron.

\section{References}

[1] E. Kuraev, E. Tomasi-Gustafsson, A. Dbeyssi, Phys. Lett. B 712, 240 (2012)

[2] A. Akhiezer, M. Rekalo, Sov.Phys.Dokl. 13, 572 (1968)

[3] A. Akhiezer, M. Rekalo, Sov. J. Part. Nucl. 4, 277 (1974)

[4] A. Bianconi, E. Tomasi-Gustafsson, Phys. Rev. Lett. 114, 232301 (2015)

[5] A. Bianconi, E. Tomasi-Gustafsson, Phys. Rev. C 93, 035201 (2016)

[6] V. Matveev, R. Muradian, A. Tavkhelidze, Lett. Nuovo Cim. 7, 719 (1973)

[7] S.J. Brodsky, G.R. Farrar, Phys. Rev. Lett. 31, 1153 (1973)

[8] R.E. Taylor, Nucleon Form Factors above 6-GeV, in Proc. of the Int. Symp. on Electron and Photon Interactions at High Energies, SLAC, 1967 (Stanford Univ., SLAC, 1967) 78-101

[9] M. Rosenbluth, Phys. Rev. 79, 615 (1950)

[10] A. Puckett, E. Brash, O. Gayou, M. Jones, L. Pentchev et al., Phys. Rev. C 85, 045203 (2012)

[11] S. Pacetti, E. Tomasi-Gustafsson, to appear in Phys. Rev. (2016). 1604.02421

[12] E. Tomasi-Gustafsson, Phys. Part. Nucl. Lett. 4, 281 (2007)

[13] J. Arrington, Phys. Rev. C 68, 034325 (2003)

[14] A.V. Gramolin, D.M. Nikolenko, Phys. Rev. C 93, 055201 (2016)

[15] L. Andivahis et al., Phys. Rev. D 50, 5491 (1994)

[16] R. Walker et al., Phys. Rev. D 49, 5671 (1994)

[17] J. Litt et al., Physics Letters B 31, 40 (1970)

[18] I. Qattan et al., Phys. Rev. Lett. 94, 142301 (2005)

[19] J. Lees et al. (BaBar Collaboration), Phys. Rev. D 87, 092005 (2013)

[20] J. Lees et al. (BaBar), Phys. Rev. D 88, 072009 (2013)

[21] E. Tomasi-Gustafsson, M. Rekalo, Phys. Lett. B 504, 291 (2001)

[22] A. Bianconi, E. Tomasi-Gustafsson, Phys. Rev. C 93, 035201 (2016)

[23] A. Bianconi, M. Radici, Phys. Rev. C 54, 3117 (1996)

[24] A. Bianconi et al., Phys. Lett. B 483, 353 (2000) 